Instituto Internacional de Investigación y Desarrollo Tecnológico Educativo INDTEC, C.A.

DOI: https://doi.org/10.29394/Scientific.issn.2542-2987.2021.6.19.16.313-328

OAI-PMH: http://www.indteca.com/ojs/index.php/Revista Scientific/oai

Ensayo Original / Original Essay

\title{
Concepción Hologógica de la Visión Docente y Políticas Educativa Venezolanas
}

\author{
Autor: César Enrique López Arrillaga \\ Unidad Educativa Nacional Bolivariana Guaicaipuro, UENBG \\ prof.cesarlopez@gmail.com \\ Caracas, Venezuela \\ https://orcid.org/0000-0002-2926-8508
}

\section{Resumen}

La Hologogía Educativa posee su fundamentación epistemológica, ontológica y axiológica en la concepción de los profesionales de la educación, el cual hace referencia de la cosmovisión que éstos perciben en su praxis educativa en los ambientes de aprendizajes, permitiendo modelar la visión del docente bajo la premisa de la Hologogía postulada por Barrera (2013); relacionada con la postura de Larrosa (2010); en referencia a la ética profesional del docente. Por otra parte, Piaget (1972); asume la inteligencia del niño desde el planteamiento de además, la comprensión de los docentes de la Educación Primaria y por último se utilizó la técnica de análisis de contenido, para la interpretación de instrumentos jurídicos y documentos oficiales del estado venezolano, el propósito de develar elementos de la percepción integral, expresada en los programas educativos de Venezuela concatenados con la pedagogía hologógica educativa y la valoración de los estudiantes como holos (mente-cuerpo-alma-espíritu) fortaleciendo al ser humano en la demanda requerida por la sociedad del siglo XXI en ilación con la comunidad planetaria.

Palabras clave: docente; escuela primaria; política educacional; percepción.

Cómo citar este ensayo:

López, C. (2021). Concepción Hologógica de la Visión Docente y Políticas Educativa Venezolanas. Revista Scientific, 6(19), 313-328, e-ISSN: 2542-2987. Recuperado de: https://doi.org/10.29394/Scientific.issn.2542-2987.2021.6.19.16.313-328

Fecha de Recepción: 21-08-2020
Fecha de Aceptación:

23-12-2020
Fecha de Publicación: 05-02-2021 


\title{
Hological Conception of the Venezuelan Teaching Vision and Educational Policies
}

\begin{abstract}
The Educational Hologogy has its epistemological, ontological and axiological foundation in the conception of education professionals, which refers to the worldview that they perceive in their educational praxis in learning environments, allowing to model the teacher's vision under the premise of the Hologogy postulated by Barrera (2013); related to the position of Larrosa (2010); in reference to the professional ethics of the teacher. On the other hand, Piaget (1972); assumes the intelligence of the child from the approach of, in addition, the understanding of the teachers of Primary Education and finally the content analysis technique was used, for the interpretation of legal instruments and official documents of the Venezuelan state, the purpose of revealing elements of the integral perception, expressed in the educational programs of Venezuela concatenated with the educational hological pedagogy and the valuation of the students as holos (mind-body-soul-spirit) strengthening the human being in the demand required by the 21 st century society in relationship with the planetary community.
\end{abstract}

Keywords: teacher; primary school; educational policy; perception.

How to cite this essay:

López, C. (2021). Hological Conception of the Venezuelan Teaching Vision and Educational Policies. Revista Scientific, 6(19), 313-328, e-ISSN: 2542-2987. Recovered from: https://doi.org/10.29394/Scientific.issn.2542-2987.2021.6.19.16.313-328

Date Received: 21-08-2020
Date Acceptance:

23-12-2020
Date Publication: 05-02-2021 


\section{Introducción}

La concepción del Docente en la Educación Primaria se encuentra en constante cambios y transformaciones en sus elementos epistémicos y axiológicos, concatenados con los factores internos considerados como los elementos que influyen en la actuación de los profesores, tales como: el desánimo, desinterés y poca motivación para realizar la labor educativa influida por los factores externos, como: crisis económica y social del Venezuela, las remuneraciones y salarios son insuficientes para los docentes, afectando su nivel adquisitivo para satisfacer sus necesidades básicas.

Además, el estado de la infraestructura de las instituciones educativas, el servicio del transporte público que dificultad el traslado de los docentes a las escuelas, aunado a ello, distancia del deber ser a la realidad que se desarrolla dista mucho del deber ser y de aquello que los miembros del contexto escolar en la cotidianidad educativa. Por lo tanto, los informantes clave consideran los factores internos y externos del contexto educativo del ejercicio de la docencia fundamentado con la realidad social desde crisis actual, que como seres humanos hace frente en su cotidianidad, lo cual afecta el desempeño de los maestros en la educación.

De acuerdo con lo anterior, la situación actual del país, aunado a ello el papel de la familia el cual deja la responsabilidad plena en la escuela. lo que ha conllevado a que muchos de los docentes se limiten solo a aplicar el currículo, en otros términos, de los planteamientos surgen nuevas situaciones dentro del contexto escolar que colocan en riesgo la formación de los estudiantes. en vista que no se están formando ciudadanos críticos, que sean responsables de sus acciones y que vengas a dar aportes para la sociedad en la cual participan.

De igual forma, la actitud de los docentes en las escuelas refleja apatía por la falta de recursos, el desinterés de la comunidad-familia-escuela; es decir, que la actitud del docente depende fundamentalmente de los factores 


\section{Ensayo Original / Original Essay}

constantes desde los externos y los internos que desmejoran el sentido de vocación y labor de los docentes.

\section{Desarrollo}

\subsection{Percepción Holística del Docente}

El docente orienta su percepción desde la visión de superar los obstáculos de la cotidianidad, con el propósito de garantizar su labor educativa en beneficio de sus estudiantes, desde una apreciación de la integralidad en la diversidad del pensamiento, realidad y vivencia de los participantes del hecho educativo.

En lo expresado anteriormente, se ilustra una noción del docente en cuanto a su identificación con respeto a su desenvolvimiento en el desarrollo de su acción pedagógica, considerando la diversidad en relación sus pensamientos, realidades de cada uno de los miembros de la comunidad posee responsabilidades inherentes al proceso de formación y aprendizaje de sus representado en la escuela.

Sin embargo, los docentes en sus reflexiones no se sienten identificados con la escuela, y están muy distantes de las familias, además en ocasiones desarrollan actividades en el aula, sin el impulso y motivación para la generación de un ambiente de aprendizaje relacionado con la integración familia-escuela, en correspondencia con lo que se viene planteando, es importante considerar los puntos de vista que poseen los docentes, un sector de los profesionales de la educación están sumergidos en una tendencia de desmotivación y apatía en la praxis cotidiana, en las aulas de clases, distantes de la práctica de principios humanísticos, separados de la creatividad, y motivación que repercute en la formación de los estudiantes.

En este orden de ideas, es evidentemente los docentes se encuentran desmotivados en el desarrollo de la acción educativo, en tal sentido solo se limitan a desarrollar contenidos, que posiblemente estén aislados de la 
realidad de los estudiantes, lo que desvirtúa la esencia de la educación en este siglo, la cual debe estar orientada a la formación de un ser activo, critico, que tome acciones dentro del contexto en el que se desenvuelve.

En este sentido, los docentes mantienen un cierto desconocimiento, lo que representa una educación basada en la integralidad de los saberes, lo cual se vienen proponiendo desde el diseño anterior al vigente en el cual se orientaba al docente no solo a relacionar las áreas de aprendizaje sino a considerar el contexto en el cual se desarrollaba el estudiante en los ambientes el proceso de enseñanza y aprendizaje de aprendizajes.

En este contexto, los docentes se orientan desde la participación de los estudiantes en su formación integral, considerando el contexto de diversas realidades de un todo, de una manera globalizada e integral, en la comprensión de la integralidad de las etapas, niveles y características en las escuelas primarias, es decir, nos es la suma de las partes sino ver la realidad educativa como la supremacía fines de la educación y la adquisición de conocimiento de forma colectiva e individual, de manera sistemática y en conjunto a los pensamientos en el marco de la percepción de la profesión concatenado con la ejecución de las actividades pedagógicas y educativas, impulsando los elementos que consoliden las facetas y etapas de los estudiantes en las actividades académicas promovidas por el docente del aula de clases.

En efecto con lo anterior, existe preeminencia del papel docente como mediador de los aprendizajes, en vista de sus aportaciones tienen un notable impacto en el desarrollo de los ciclos de vida de los estudiantes. es evidente que la vocación y entusiasmo de esta profesional marca una pauta muy importante en lo referente a la integralidad y calidad de los aprendizajes. En este orden de ideas, se observa una apreciación los maestros activos en el sistema educativo se consideran como orientadores que refuerzan los valores y principios en el marco de la responsabilidad en la ejecución de las áreas 


\section{Ensayo Original / Original Essay}

académicas en los estudiantes reconociéndolos desde sus individualidades, particularidades con relación a la realidad cognitiva, social y espiritual que afecta su formación en la construcción e intercambio de saberes, conocimientos y emociones.

Cabe destacar, los docentes se consideran responsables de promover en las aulas de clases un ambiente armonioso, agradable a través de estrategias didácticas las cuales fortalezcan los aprendizajes significativos desde la construcción colectiva de los conocimientos en los estudiantes, de allí que, como seres humanos en formación, tendrán un ritmo e intereses propios en el proceso educativo integral y holístico, desarrollado mediante la participación del educador

En el mismo orden, se evidencia una comprensión de los estudiantes como parte de un trabajo integral desde las necesidades de los estudiantes desde su diversidad, con la intención de planificar las acciones necesarias para la formación integral con la consideración de cada estudiante emprende un proceso único de enseñanza y aprendizaje propio de sus intereses, de allí que la acción pedagógica surge de su forma de pensar y actuar de los educadores como elemento esencial para una práctica educativa integral.

Por lo tanto, en la actualidad prevalece una tendencia de docentes que mantienen su percepción de su rol relacionado con buen trato desde el amor, la tolerancia. En este orden y dirección, el docente de hoy es creativo, innovador desde la acción educativa orientada en el humanismo, innovador, impartir los valores para la promoción de un ambiente armonioso, sano y un ambiente escolar agradable.

Dentro de este marco, los valores y principios del docente se incluyen en su personalidad y concepción hologógica de desempeñarse en relaciones humanas desde los procesos de enseñanzas y aprendizajes con dedicación y compromiso con sus estudiantes, en este contexto cabe señalar a Larrosa (2010): "inclinación natural para dedicarse a la actividad profesional de 
enseñar con entusiasmo, compromiso y confianza en el poder de la educación, dedicación especial y de servicio hacia los demás" (pág. 49).

En este orden, el docente se evidencia que relaciona su vida, su cotidianidad con el ejercicio de la profesión, agrega también la identificación que cada profesor debe tener, es vocación hacia aquello que hace, conocimientos mediante un proceso desde la Hologogía, reconociendo la diversidad de cada niño, niña y adolescentes. Por ende, el fortalecimiento del área espiritual de los niños en función de propiciar la sensibilidad humana desde la aceptación de diferencias y tolerancia en el aula, como principio básico de la convivencia e intercambio de saberes y conocimientos desde cada cual.

En consecuencia, posee una visión del docente observando una apreciación del contexto social en se encuentran los estudiantes dirige la labor docente al impulso de los aprendizajes y conocimientos para satisfacer las necesidades de los educandos, se relaciona con la develadas por los docentes relacionada con la perspectiva de la vocación sobre su manera de tratarse y tratar a los otros, por consiguiente, el docente ejerce su rol en el ambiente de aprendizaje es un ambiente abierto dinámico y humano.

En lo señalado anteriormente, se muestra el docente con la percepción desde el desarrollo de la labor educativa orientada la praxis cotidiana en las aulas y entorno escolar, además, relacionada con la perspectiva integral del actuar y el deber ser, del proceso de aprendizaje y enseñanza, consolidando los valores como: la tolerancia, solidaridad y respetado mutuo en los estudiantes, cumpliendo su papel de formador de educandos, que se consoliden como ciudadanos comprometidos, democráticos, participativos con el contorno social.

Entonces en otras palabras, la concepción se orienta en apreciar y comprender los estudiantes en continuo aprendizaje en el marco de sus necesidades, transcender su visión en labor educativa desarrollando la 
motivación, dedicación y responsabilidad con la formación hologógica de cada educando, desarrollando la integralidad de ser social en el marco de sus creencias, vivencias, hábitos y preceptos de pensamientos hilado con las perspectivas, de la forma de pensar de los docentes desde su vivencias, experiencias y conocimientos.

En virtud de lo planteado, la concepción hologógica un grupo de docentes entrevistados manifiestan desanimo y apatía para asistir a clases, influenciados la situación problemática que atraviesa Venezuela, en ocasiones se encuentra apático en las actividades de la escuela, así mismo se relejan en los testimonios sobre la concepción de un docente tradicionalista que no permite incrementar su creatividad solo apegándose a estrictamente lo contemplado en el currículo de educación. Tomando en cuenta, la postura de Piaget (1972):

El docente debe ser un guía y orientador del proceso de enseñanza y aprendizaje, él por su formación y experiencia conoce que habilidades requerirles a los alumnos según el nivel en que se desempeñe, para ello deben plantearles distintas situaciones problemáticas que los perturben y desequilibren (pág. 145).

De acuerdo a lo anterior, se relaciona con la concepción hologógica orientada a la labor educativa del docente y forma de ver las realidades, contextos y ambientes, desde la amplitud y la diversidad, que posee en su acción pedagógica como orientador, facilitador entendiendo su rol y practica educativa en la promoción de estrategias didácticas significativas, valores y principios morales que generen un ambiente agradable en los estudiantes mediante la unificación de los actores del quehacer educativo, reconociendo a los estudiantes sus individualidades, necesidades e interés en el proceso de enseñanza y aprendizaje, que garantice su formación integral en la escuela primaria. En relación con Barrera (2013a):

La comprensión hologógica implica la visión de la persona- 


\section{Ensayo Original / Original Essay}

principio, centro y fin de la educación, como una integridad, como una realidad, íntegra, integral y a su vez trascendente. A diferencia de las posturas antropológicas que fraccionan y atomizan la condición humana, la educación holística insiste en la necesidad de apreciar la persona en su totalidad, en su conjunto, desde la integralidad, como complejidad, pues apreciadas las cosas desde la totalidad se puede percibir con más precisión los detalles propios de su condición humana (pág. 53).

Partiendo de las afirmaciones anteriores, la Concepción Hologógica que emergió, considera la educación con una visión de la aplicación de estrategias pedagógicas, considerando a los alumnos con el ritmo propio de aprender y conocer desde una percepción amorosa y tolerante desde la diversidad de personalidad en un entorno escolar.

\subsection{Visión del Docente}

Al hacer referencia a la visión del docente se hace mención al pensamiento y Concepción hologógica que posee en cuanto a las realidades, hechos, vivencias, experiencias y sentimientos frente a su praxis educativa, desde su comprensión mental, siendo percibido por el quehacer educativo desde la perspectiva del ideario y fines de la educación, en lo que están involucrados los informantes claves del quehacer educativo, proyectándose el docente como aquel posee la visión del actuar y pensar dando paso a la aplicación de actividades académicas.

Es importante resaltar los cambios en los alumnos, una subcategoría emerge con los informantes claves que expresan como se han observado las perspectivas de las diversas formas de percibir las realidades en la cotidianidad, que conforman la visión del docente, por ende, los docentes en la actualidad se encuentran con esperanzas y amor por su labor a pesar de todo los problemas que atraviesa, aún continúan con su amor al servicio y por sus estudiantes, es verdad que a veces hay apatía y poco ánimo, dado lo que 
se está viviendo la crisis país, sin embargo, aún hay docentes comprometidos con el futuro del país, con la formación de venezolanos y venezolanas.

De acuerdo con las ideas anteriores, los docentes proyectan un compromiso con la labor que desarrollan en cuanto llevan a cabo una función en la que se evidencia amor hacia el trabajo que hacen. no obstante, hay un grupo que se encuentran desmotivado por la situación que impera en el contexto social venezolano lo cual atenta con lo que sería el futuro formativo de los estudiantes y por ende del país, si no se cumple una función integral, desde la mirada del informante, se está frente a dos grupos de docentes uno que trabaja limitado por la situación del contexto que no le permite el desarrollo de una labor de manera integral y otro grupo que si está en el desarrollo de las políticas educativas.

Son orientadas a una función de calidad, en la que se trabaje con calidad educativa, por ello, la percepción del docente se concatena con la educación de forma integral atendiendo todos los aspectos de la enseñanza y aprendizajes, comprendiendo a cada estudiante en su ritmo de aprender, su entorno familiar y social, es difícil porque siempre tenemos los casos de que la familia no apoya, el docente debe tener la perspectiva de brindar el soporte pedagógico y académico, pero en algunos momentos transcender hasta lo espiritual, con un consejo o una charla de reflexión en los ambientes del aprendizaje.

Por otra parte, los docentes en su concepción de la atención de los estudiantes, desde los principios y valores orientados a la comprensión hologógica de los educandos, regente a sus características y personalidades únicas y diversas como seres humanos, deben plantearse una visión humana del proceso educativo de forma integral que reúna todos los actores educativos. En relación con las anteriores implicaciones, Barrera (2013b): sostiene que "atender el quehacer educativo desde una comprensión holista, integral, integrativa y universal, implica el acercamiento a una visión 
igualmente integrativa y holista del ser humano" (pág. 24).

Además, se hace coincidente la visión de los profesionales de la educación al expresar aquello que desean en cuanto a comprender las diversas realidades de los estudiantes en el contexto escolar, formar ciudadanos con valores relacionados con el amor, tolerancia, es decir, desde la integralidad y complejidad de la realidad educativa, de igual forma, los docentes se ven expuesto a la apatía y el desamino de asistir a la escuela y de atender a los estudiantes, relacionado con la crisis actual que atraviesa el país, que afecta la actuación del docente.

En este mismo orden, existe un grupo de docente dispuesto a mantener sus posiciones y postura desde la mirada de una visión de sus pensamientos, para vencer los obstáculos y barreras, transcender lo académico, lo pedagógico e internalizan su misión en atender el estudiante desde sus propias características y necesidades, formando un Ser Social, que sean participes de la transformación que se desarrolla en la sociedad actual, la cual se relaciona con la concepción de los docentes. El Estado en sus Políticas Educativas manifestadas con una visión desde la función como ente rector, en que cada cual se desarrolla en el aprender, ser, hacer y convivir en el proceso de enseñanza y aprendizaje.

De allí que la visión de los docentes está orientada a la percepción de ellos con la realidad individual en la labor educativa diaria, es continua, reflexiva e interdependiente dentro de un proceso personal, basada en una concepción previa que reúne las experiencias y vivencias de cada docente, en las experiencias de la labor de formar los niños, mediante la promoción del amor y la humildad se concreta una educación para todos siempre tener presente que los estudiantes son un mundo cada uno, que son diferentes, tienen su propio ritmo de aprender, porque se necesita cultivar la paciencia para guiarlos en el camino del aprendizaje de los estudiantes en su proceso formativo, considerando su propio ritmo de aprendizaje. 
Es importante destacar, algunos aspectos de la visión del docente en las aulas de clases, se materialice su labor formativa contextualizada con la realidad del estudiante, desde una concepción de humanidad y amor que se integran en la pedagogía del docente, en función de percibir las realidades del entorno escolar de forma amplia e integral como guía de aprendizajes de los estudiantes, los cuales proyectarán en el entorno y la formación como ciudadanos más democráticos, responsables y participativos, desde las voces de los informantes surge un visión integral, espiritual, amorosa, humildad desde la concepción flexible que integré todos los pensamientos, sentimientos y forma de actuar de nosotros, en la búsqueda de una labor educativa desde la integridad, espiritualidad, diversidad, amor y para los estudiantes.

\subsection{Interpretación Critica: Actuación del Docente desde la mirada holística en el marco de los programas educativos del Estado venezolano}

Mediante la interpretación de los elementos de la actuación y concepción a través de su cotidianidad educativa en las aulas de clases, quien posee la responsabilidad de materializar los programas educativos tales como: Canaima educativa, colección bicentenaria, el programa de alimentación escolar, el bolso y uniformes escolares, sin embargo, se observan diversas visiones desde la praxis educativa relacionada con la educación integral, como se puede apreciar en el cuadro 1.

Cuadro 1. Interpretación crítica de la actuación del docente y los programas educativos del Estado venezolano.

\begin{tabular}{|c|c|}
\hline Documento & Análisis \\
\hline $\begin{array}{c}\text { Constitución de la República } \\
\text { Bolivariana de Venezuela } \\
\text { (1999) }\end{array}$ & $\begin{array}{l}\text { En el marco de los principios constitucionales en los } \\
\text { artículos } 102 \text { y } 103 \text {, se prescribe la formación de } \\
\text { ciudadanos críticos, reflexivos, participativos, } \\
\text { democráticos en el marco de los valores de la } \\
\text { solidaridad, autodeterminación, la soberanía } \\
\text { concatenado con el artículo } 6 \text { de la ley in comento. } \\
\text { Al mismo tiempo, concreta el carácter constitucional } \\
\text { a la educación como un derecho humano } \\
\text { intransferible, obligatorio y gratuito hasta la etapa }\end{array}$ \\
\hline
\end{tabular}


Instituto Internacional de Investigación y Desarrollo Tecnológico Educativo INDTEC, C.A.

DOI: https://doi.org/10.29394/Scientific.issn.2542-2987.2021.6.19.16.313-328

OAI-PMH: http://www.indteca.com/ojs/index.php/Revista Scientific/oai

Ensayo Original / Original Essay

Ley Orgánica de Educación

(2009) media y diversificada (secundaria). En este sentido, la Carta Magna cumple con los principios y garantías constitucionales de protección de los ciudadanos en sus derechos humanos.

La presente ley rige los principios y valores del sistema educativo y asigna a cada uno de los actores que se encuentran involucrados su deberes y derechos. En el artículo 1, se evidencia la supremacía de la importancia de la educación por parte de Venezuela, principalmente garantiza la igualdad género (art. 3) y la práctica de la equidad e inclusión (art. 3) por lo que se puede observar el ente rector, quien promueve las políticas y programas a las zonas educativas, municipios escolares y escuelas. Por otro lado, la ley preceptúa el Estado Docente con funciones rectoras en la educación (art. $5)$.

Fuente: El Autor (2019).

Con el análisis de contenido realizado, se le atribuye al Estado la responsabilidad de generar los procesos dinámicos en el sistema educativo, que correspondan a las necesidades y capacidades de los actores del quehacer educativo. Igualmente, promueve una concepción integral de la educación relacionada con los objetivos de los planes, programas, proyectos de desarrollo socioeconómico y político de la nación.

De allí que, surge la importancia del marco jurídico relacionado con el ámbito educativo como garantía de los derechos constitucionales y derechos humanos tan relevantes como el de la educación. Se evidencia los elementos de una concepción sobre la educación en el marco de continuo humano propiciando la educación bolivariana por etapas de vida y niveles de educación en un espiral considerado una educación holística e integral, que converger: Hacer, Ser, Convivir y Aprender, desde una perspectiva transdisciplinaria que estimula la creativa del Ser Social.

Asimismo, la educación integral desde la práctica humanista en el aula, entendiendo a los estudiantes como sujetos activos de su propio aprendizaje y formación durante su desarrollo humano, esto es, la integralidad y unicidad 
del Ser desde la mirada concreta en la acción pedagógica. todo se concreta, conduce a una integración de saberes considerados desde la cotidianidad y todo aquello que influye la formación de los estudiantes lo que se traduce en una formación social e inclusiva, partiendo de la concepción que se reflejan en las leyes y políticas educativas del siglo XXI.

\section{Conclusiones}

Los puntos de vista de los docentes se dirigen la comprensión de la integralidad y diversidad de eventos que surgen de la realidad y hechos del quehacer educativo relacionado con el pensamiento y percepción de los maestros. En este sentido, el pensamiento y perspectivas en la Concepción del docente debe prevalecer la interpretación con la formación integral en los estudiantes como seres participativos, críticos, reflexivos, auténticos con procesos de aprendizajes de forma individual y autentica, partiendo de las diversas interacciones para la construcción del conocimiento desde sus saberes propios en forma colectiva desde la mirada de los informantes claves.

Los docentes mantienen una visión hologógica que se orienta en la percepción del todo, como proceso integrador de realidades en el contexto educativo, en el marco del proceso social, económico, político y cultural que se desarrolla en la sociedad, las perspectivas y visiones en cuanto al desarrollo de los objetivos, propósitos y acción educativa de los docentes en las aulas de clases.

\section{Referencias}

Barrera, M. (2013a,b). Hologogía. Introducción a la educación holística. Tercera Edición. Caracas, Venezuela: Ediciones Quirón-Sypal. Constitución de la República Bolivariana de Venezuela (1999). Gaceta Oficial

Extraordinaria N. ${ }^{\circ} 36.860,30$ de diciembre. Caracas, Venezuela:

Asamblea Nacional Constituyente. 
Larrosa, F. (2010). Vocación docente versus profesión docente en las organizaciones educativas. Revista Electrónica Interuniversitaria de Formación del Profesorado, 13(4), 43-51, e-ISSN: 1575-0965. Recuperado de: https://www.redalyc.org/articulo.oa?id=217015570004 Ley Orgánica de Educación (2009). Gaceta Oficial No 5.929 Extraordinario del 15 de agosto. República Bolivariana de Venezuela: La Asamblea Nacional.

Piaget, J. (1972). El nacimiento de la inteligencia en el niño. Madrid, España: Editorial Aguilar. 


\section{César Enrique López Arrillaga}

e-mail: prof.cesarlopez@gmail.com

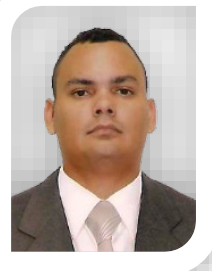

Nacido en La Guaira, estado La Guaria, Venezuela, el 7 de mayo del año 1985. Doctor en Ciencias de la Educación por la Universidad Latinoamericana y del Caribe (ULAC); Magister Scientiarum en Educación Superior de la Universidad Nacional Experimental de la Fuerza Armada (UNEFA); Licenciado en Educación de la Universidad Nacional Experimental Simón Rodríguez (UNESR); Comisionado Municipal de la Oficina Antidrogas del municipio Independencia; Director de Recursos Humanos de la Alcaldía del municipio Independencia; Jefe de Personal de la Policía Municipal Independencia; Director de Administración, Finanzas y Presupuesto de la Contraloría del municipio Simón Bolívar; Tutor de Trabajos Especiales de Grado, Especializaciones y Maestrías en la Universidad Pedagógica Experimental Libertador (UPEL) y la UNEFA; Docente de Educación Media General en la Unidad Educativa Nacional 28 de Marzo; Coordinador de Control de Estudios de la Unidad Educativa Privada Coronel Antonio Nicolas Briceño; Actualmente docente de Educación Primaria de la Unidad Educativa Nacional Bolivariana Guaicaipuro; Arbitro Calificado y Comité Académico Científico de la Revista Scientific (e-ISSN: 2542-2987); Arbitro de la Revista Ensayos Pedagógicos (e-ISSN: 2215-3330); y de la Revista Pedagogía y Saberes (e-ISSN: 2500-6436) de la Facultad de Educación de la Universidad Pedagógica Nacional (UPN), Colombia.

El contenido de este manuscrito se difunde bajo una Licencia de Creative Commons ReconocimientoNoComercial-Compartirlgual 4.0 Internacional 